\title{
A Single Institution's Experience with Cytogenetic and MRD Outcomes in Pediatric Acute Lymphoblastic Leukemia
}

\author{
Asim Amjad, Rabia Muhammad Wali, Sadia Anjum and Raheela Mansoor
}

\begin{abstract}
Objective: To determine the frequency of cytogenetic type and its significance in the prognostic outcome of the pediatric patients in acute lymphoblastic leukemia (ALL), aged 1 to 15 years, and also determine the importance of minimal residual disease (MRD) in the management of the condition.

Study Design: An observational study.

Place and Duration of Study: Pediatric Oncology Ward, Shaukat Khanum Cancer Hospital, Lahore, from January 2015 to July 2017.

Methodology: Patients aged 1-15 years, diagnosed with ALL, were included. Studied variables were cytogenetic type and MRD outcome in patients with ALL. Patients under one year of age and more than 15 years, or those having comorbidities, were excluded.

Results: Total 150 patients' data were retrieved from the Hospital database. One hundred and thirty-three belonged to age 1 to 5 years group $(89 \%)$ and $17(11 \%)$ were in 5 to 10 years group. The mean age of the patient was $4.3 \pm 3.1$ years. One hundred and two (68\%) were males; whereas, $48(32 \%)$ were females. Pre B acute lymphoblastic leukemia was diagnosed in $139(93 \%)$ patients and $11(7 \%)$ were diagnosed with Pre T acute lymphoblastic leukemia. Standard risk was observed in $120(80 \%)$ patients and $30(20 \%)$ patients were on high risk as per National Cancer Institute (NCI) Guidelines. Regimen A was used in 125 (83.3\%), Regimen B in 16 (10.7\%), and Regimen C in 9 (6\%) patients. BCR-ABL was positive in 2 (1.30\%), TEL-AML in 68 (45\%), MLL in 5 (3.30\%), and normal in 54 (36\%). MRD at day 29 was negative in 40 (93\%) and positive in $3(7 \%)$. The karyotyping was done in $128(85 \%)$ patients, out of which $68(53 \%)$ were hyperploids, 41 (32\%) euploid, and $19(15 \%)$ were hypoploid. Death was observed in $22(15 \%)$ patients. Nineteen (86\%) deaths were due to fungal and bacterial sepsis; and disease-related deaths were noted in $3(14 \%)$ patients.

Conclusion: The role of MRD and cytogenetics in risk assessment has improved in the early prognosis determination.
\end{abstract}

Key Words: Cytogenetics, Acute lymphoblastic leukemia (ALL), Minimal residual disease (MRD).

\section{INTRODUCTION}

Acute lymphoblastic leukemia (ALL) is among one of the most common hematological malignancies seen in children. Children with acute leukemia mostly present with pallor, bone pain, bleeding manifestations, and hepatosplenomegaly. 1,2 In the last three decades, many changes were made in treatment and outcomes of acute lymphoblastic leukemia patients, and at the start of the $21^{\text {st }}$ century almost $80 \%$ of patients were expected to survive without relapse after first-line treatment.3-6 Factually, the treatment intensity received by a patient with acute lymphoblastic leukemia was relapse risk-based, which was predicted by a combination of clinical, cytogenetic, and morphological early response criteria.3,5,6 Cytogenetics and minimal residual disease (MRD) is of great importance in the prediction of outcome in acute lymphoblastic leukemia patients. ${ }^{7,8}$ The $t(9 ; 22)(q 34 ; q 11.2)$

Department of Pediatric Oncology, Shaukat Khanum Memorial Cancer Hospital, Johor Town, Lahore, Pakistan

Correspondence: Dr. Asim Amjad, Department of Pediatric Oncology, Shaukat Khanum Memorial Cancer Hospital,

Johor Town, Lahore, Pakistan

E-mail: amjadasim74@gmail.com

Received: July 20, 2018; Accepted: November 29, 2018 chromosomal translocation, i.e. the Philadelphia (Ph.) chromosome, and the resulting breakpoint cluster regionAbelson murine leukemia viral oncogene homolog 1 (BCR-ABL1) fusion protein1 occur in 20 to $30 \%$ of adult patients with acute lymphoblastic leukemia (ALL) and in 3 to $5 \%$ of patients with childhood ALL, because of its high rate of relapse with chemo-therapy. 9,10

Over the past decade, tyrosine kinase inhibitors (TKIs) targeting BCR-ABL1 have been added to chemotherapy regimens; a Children Oncology Group (COG) showed that combined Imatinib with intensive post-remission induction chemotherapy yielded a significant improvement in event-free survival (EFS) rates for childhood Ph.-positive ALL.

The primary objective of this study was to see the different cytogenetic abnormalities in Pakistani population-based on karyotyping and fluorescent in situ hybridisation (FISH) analysis and its significance in the prognostic outcome of the pediatric patients in ALL aged 1 to 15 years. The secondary objective was to see the results of MRD and its significance in the patients at the end of induction.

\section{METHODOLOGY}

The study design was observational retrospective. The site of the study was Department of Pediatric Oncology, 
Shaukat Khanum Memorial Cancer Hospital, Lahore. Information was retrieved from all patients registered in Pediatric Oncology Department from January 2015 to July 2017, after taking International Research Board (IRB) approval. The study enrolled all the patients aged 1-15 years who were diagnosed with acute lymphoblastic leukemia. Exclusion criteria were age less than 1 year and greater than 15 years or the patient with comorbidities. Studied variables were cytogenetic type and MRD outcome in patients with ALL. Since the study centre started doing MRD in January 2017, so the sample for MRD included patients from January 2017 till July 2017. Patients were categorised according to National Cancer Institute $(\mathrm{NCl})$ risk stratification on the basis of age, WBC count, phenotype, and karyotyping. All patients were diagnosed on flow cytometry either on bone marrow or peripheral blood at baseline. Cytogenetic and karyotyping was also done at baseline. High risk karyotyping and cytogenetic were defined as hypoploidy, MLL and BCR-ABL translocations. Bone marrow biopsy was done at day-8, day-15 and day-29 at the end of induction. Bone marrow in remission was defined as bone marrow having less than $5 \%$ blast cells on examination. Additional testing at day 29 was done on bone marrow in the form of minimal residual disease (MRD), which can detect even few microscopic blast cells and its normal value was less than $0.001 \%$.

Protocol used for treatment was UKALL interim guidelines 2013. All cases received either regimen A with 3 drugs induction (vincristine, dexamethasone, pegylated asparagine) or regimen $B$ and $C$ in induction with 4 drugs (vincristine, dexamethasone, pegylated asparagine, donorubicin) on the basis of their risk stratification. The MLL gene abnormalities and hypoploidy was treated with regimen $C$ because of its high risk stratification. The patients having BCR-ABL translocations were treated with the combination of tyrosine kinase inhibitors due to the better survival rates with combination therapy. Patients who had either bone marrow not in remission or positive MRD post induction were escalated according to UKALL Interim Guidelines 2013 for ALL.

All the collected data were formalised electronically and analysed later by using Microsoft Excel version 10 and SPSS version 19. Descriptive statistics were applied to calculate mean and standard deviation for quantitative variables like age. Frequency distribution and percentages were calculated for qualitative variables like gender, cytogenetic type, MRD findings and remission.

\section{RESULTS}

One hundred and fifty patients' data were retrieved from the Hospital database, out of whom $133(89 \%)$ children belonged to age 1 to 5 group; whereas, 17 (11\%) were in 5 to 10 years age group. The mean age of the patients was 4.3 years. Most of the patients were males $(n=102$,
Table I: Clinical and diagnostic findings in children with ALL.

\begin{tabular}{l|r|c}
\hline Findings & $\mathrm{n}$ & $\%$ \\
\hline MRD at day 29 & 43 & $29 \%$ \\
Performed in patients & 40 & $93 \%$ \\
Negative & 3 & $7 \%$ \\
Positive & & \\
\hline FISH & 2 & $1.30 \%$ \\
BCR-ABL & 68 & $45.30 \%$ \\
TEL-AML & 5 & $3.30 \%$ \\
MLL & 54 & $36 \%$ \\
NORMAL & 21 & $14.10 \%$ \\
Not done & 125 & $83 \%$ \\
\hline Bone marrow remission at day 15 & 20 & $13 \%$ \\
Yes & 5 & $3 \%$ \\
No & & \\
Not done & 142 & $95 \%$ \\
Bone marrow remission at day 29 & 5 & $3 \%$ \\
Yes & 3 & $2 \%$ \\
No & & \\
Not done & & \\
\hline
\end{tabular}

$67 \%)$; whereas, $48(33 \%)$ were female. Pre B acute lymphoblastic leukemia was seen in 139 (93\%) and $11(7 \%)$ had, Pre T ALL. Standard risk was in $120(80 \%)$ patients and $30(20 \%)$ patients were categorised high, risk as per National Cancer Institute $(\mathrm{NCl})$ guidelines. Regimen A treatment was given in $125(83 \%)$ patients, Regimen B in $16(11 \%)$, and Regimen C in $9(6 \%)$. FISH showed BCR-ABL in $2(1.30 \%)$, TEL-AML in $68(45 \%)$, MLL in $5(3 \%)$, normal in $54(36 \%)$ and not done in 21 $(14 \%)$ due to its unavailability at that time. MRD at day-29 was done in 43 patients, which was negative in 40 (93\%) and positive in $3(7 \%)$. Karyotyping was done in 128 $(85 \%)$ out of which $68(53 \%)$ were hyperpoild, $41(32 \%)$ euploid and 19 (15\%) were hypoploid.

Death was seen in 22 (15\%) patients (Table I). Total 19 $(86 \%)$ deaths were due to fungal and bacterial infections leading to sepsis. Disease related deaths were noted in $3(14 \%)$ patients only. All patients were of Pre B acute lymphoblastic leukemia phenotype standard risk as per National cancer institute $(\mathrm{NCl})$ criteria, and there was no patient with hypoploidy, and high risk MLL, BCR-ABL cytogenetic. There were total 3 disease related deaths out of which minimal residual disease was positive in 2 $(67 \%)$ at the end of induction, and $1(33 \%)$ patient had day-29 bone marrow positive with blast cells had not achieved remission. Out of these, three disease related deaths, two patients $(67 \%)$ patients were escalated to Regimen C due to induction failure, however, 1 (33\%) patient died of central nervous system relapse at maintenance therapy.

\section{DISCUSSION}

This study was done to highlight the Institutional experience with Cytogenetic and MRD outcomes in acute lymphoblastic leukemia pediatric patients. These results not only showed the profiling of patients with acute 
lymphoblastic leukemia in children like risk stratification, cytogenetic type, and assigned treatment accordingly, but also identified that the low risk of relapse can be achieved by rapid clearance of MRD at the end of induction therapy. This finding is supported by other published studies. 3,10

The incidence of relapse was low in comparison with the previous studies.3,11 The significance of the rapid morphological clearance (RMC) of blasts in peripheral blood (PB) and bone marrow (BM) during the initial levels of chemotherapy was demonstrated by various published studies. ${ }^{12,13}$ Early in therapy the assessment of RMC at different times was used as a tool for risk group provision in acute lymphoblastic leukemia therapy. 14,15

This method has recently been replaced by minimal residual disease (MRD) assessment for the identification of the risk group. A poor prognosis was observed among patients who failed after therapy induction in achieving the morphological remission. Somewhat dismal outcomes were not observed in all these patients, and due to the induction remission therapy duration variance among different studies, it is difficult to compare the outcomes among them. ${ }^{14}$

Often high-risk features were observed among induction failure patients; these features include $T$ cell immune phenotype, MLL-rearrangement (MLL-r), BCR-ABL translocation, age, and higher WBC count. The finding of our study showed high percentages of TEL-AML. This finding is supported by other available published studies. ${ }^{15,16}$ The other significant result of our study was the large number of children were under 5 years of age. Hence the TEL-AML was the commonest cytogenetic under 5 years of age children. The worse outcome was highly correlated to the presence of residual leukemia in BM on 8th day of induction or some time 15th day. ${ }^{17}$

Without any shadow of doubt, nowadays MRD is considered to be the most powerful prognostic factor in childhood acute lymphoblastic leukemia.18 The sensitivity and specificity of MRD has been assessed by many studies at various time points with variety of therapy protocols by using PCR-based technology. ${ }^{19-20}$ Hence, the utility of MRD in prognosis determination of de novo acute lymphoblastic leukemia patients for first relapse and in re-induction of chemotherapy is very crucial and vital. ${ }^{13}$ Other findings of our study include, protocols with regimen $A$ as most commonly observed in the present study population.

This study is only one of its own kind in the region, so more studies in the domain will definitely help in improving the therapy success rate and protocol development in acute lymphoblastic leukemia patients. There was a limitation of this study because of the unavailability of MRD before January 2017 at the study centre, so it was not done in all the patients included in this study. However, sample size was adequate to have a concrete evidence about the prognostic significance of MRD in pediatric leukemic patients.

\section{CONCLUSION}

Cytogenetics and MRD are imperative in risk stratification of a patient with acute lymphoblastic leukemia. An accurate risk stratification of a patient, with acute lymphoblastic leukemia, guides about the type of chemotherapy regimen to be given; and this in turn, reduces the risk of disease relapse due to the perfect choice of treatment. This is also helpful in preventing the undue administration of intensive chemotherapy drugs to the patients, and thus preventing them from chemotherapy-related toxicities and deaths.

\section{REFERENCES}

1. Bönig H, Yong-Mi K. VLA4 in acute lymphoblastic leukemia. Targeted therapy of acute myeloid leukemia 2014; 637-54.

2. Morales-Sanchez, Abigail, Ezequiel M. Infectious etiology of childhood acute lymphoblastic leukemia, hypotheses and evidence. Clinical epidemiology of acute lymphoblastic leukemia - From the molecules to the clinic, 2013.

3. Mitchell C, Payne J, Wade R, Vora A, Kinsey S, Richards S, et al. The impact of risk stratification by early bone-marrow response in childhood lymphoblastic leukemia: Results from the United Kingdom Medical Research Council trial ALL97 and ALL97/99. Br J Haematol 2009; 146:424-36.

4. Moghrabi A, Levy DE, Asselin B, Barr R, Clavell L, Hurwitz C, et al. Results of the Dana-Farber cancer institute ALL consortium protocol 95-01 for children with acute lymphoblastic leukemia. Blood 2007; 109: 896-904.

5. Möricke A, Reiter A, Zimmermann M, Gadner H, Stanulla M, Dördelmann $\mathrm{M}$, et al. Risk-adjusted therapy of acute lymphoblastic leukemia can decrease treatment burden and improve survival: Treatment results of 2169 unselected pediatric and adolescent patients enrolled in the trial ALL-BFM 95. Blood 2008; 111:4477-89.

6. Jeha S, Coustan-Smith E, Pei D, Sandlund JT, Rubnitz JE, Howard SC, et al. Impact of tyrosine kinase inhibitors on minimal residual disease and outcome in childhood Philadelphia chromosome-positive acute lymphoblastic leukemia. Cancer 2014; 120:1514-9.

7. Pui $\mathrm{CH}$, Sandlund JT, Pei D, Campana D, Rivera GK, Ribeiro RC, et al. Improved outcome for children with acute lymphoblastic leukemia: Results of Total Therapy Study XIIIB at St Jude Children's Research Hospital. Blood 2004; 104:2690-6.

8. Cavé $\mathrm{H}$, van der Werff ten Bosch J, Suciu S, Guidal C, Waterkeyn $\mathrm{C}$, Otten $\mathrm{J}$, et al, Clinical significance of minimal residual disease in childhood acute lymphoblastic leukemia. European Organization for Research and Treatment of Cancer Childhood Leukemia Cooperative Group. N Engl J Med 1998; 339:591-8.

9. van Dongen JJ, Seriu T, Panzer-Grümayer ER, Biondi A, Pongers-Willemse MJ, Corral $\mathrm{L}$, et al. Prognostic value of minimal residual disease in acute lymphoblastic leukaemia in childhood. Lancet 1998; 352:1731-8.

10. Conter V, Bartram CR, Valsecchi MG, Schrauder A, PanzerGrümayer R, Möricke A, et al. Molecular response to treatment 
redefines all prognostic factors in children and adolescents with B-cell precursor acute lymphoblastic leukemia: Results in 3184 patients of the AIEOP-BFM ALL 2000 study. Blood 2010; 115:3206-14

11. Pui CH, Campana D, Pei D, Bowman WP, Sandlund JT, Kaste SC, et al. Treating childhood acute lymphoblastic leukemia without cranial irradiation. N Engl J Med 2009; 360:2730-41.

12. Vora A, Wade R, Mitchell CD, Goulden N, Richards S. Improved outcome for children and young adults with T-cell acute lymphoblastic leukaemia (ALL): Results of the United Kingdom Medical Research Council (MRC) Trial UKALL 2003. Blood 2008; 112:908.

13. Miller DR, Leikin S, Albo V, Sather H, Karon M, Hammond D. Prognostic factors and therapy in acute lymphoblastic leukemia of childhood: CCG-141. A report from childrens cancer study group. Cancer 1983; 51:1041-9.

14. Campana D. Minimal residual disease monitoring in childhood acute lymphoblastic leukemia. Curr Opin Hematol 2012; 19: 313-8.

15. Schultz KR, Pullen DJ, Sather HN, Shuster JJ, Devidas M, Borowitz MJ, et al. Risk- and response-based classification of childhood B-precursor acute lymphoblastic leukemia: A combined analysis of prognostic markers from the Pediatric
Oncology Group (POG) and Children's Cancer Group (CCG). Blood 2007; 109:926-35.

16. Lauten M, Möricke A, Beier R, Zimmermann M, Stanulla M, Meissner $\mathrm{B}$, et al. Prediction of outcome by early bone marrow response in childhood acute lymphoblastic leukemia treated in the ALL-BFM 95 trial: Differential effects in precursor B-cell and T-cell leukemia. Haematologica 2012; 97:1048-56.

17. Schrappe M, Hunger SP, Pui CH, Saha V, Gaynon PS, Baruchel A, et al. Outcomes after induction failure in childhood acute lymphoblastic leukemia. N Engl J Med 2012; 366:1371-81.

18. Nachman JB, Heerema NA, Sather H, Camitta B, Forestier E, Harrison CJ, et al. Outcome of treatment in children with hypodiploid acute lymphoblastic leukemia. Blood 2007; 110:1112-5.

19. Borowitz MJ, Devidas M, Hunger SP, Bowman WP, Carroll AJ, Carroll WL, et al. Clinical significance of minimal residual disease in childhood acute lymphoblastic leukemia and its relationship to other prognostic factors: A Children's Oncology Group study. Blood 2008; 111:5477-85.

20. Coustan-Smith E, Behm FG, Sanchez J, Boyett JM, Hancock ML, Raimondi SC, et al. Immunological detection of minimal residual disease in children with acute lymphoblastic leukaemia. Lancet 1998; 351:550-4. 